\title{
Massive thyroid gland metastasis from non small cell lung cancer
}

\begin{abstract}
Thyroid gland metastasis is rarely observed in clinical practice. In this report, we describe a case of a patient with primary lung cancer who developed massive metastasis to the thyroid over a short period. Because of rapid tumor growth, we there was a misdiagnosis between lymphoma, anaplastic and hypo pharyngeal cancer and lastly metastatic lesion.
\end{abstract}

Keywords: lung cancer, metastasis to thyroid, fine needle aspiration cytology, thyroid ultrasound

\author{
Special Issue - 2018
}

\author{
Diana Simoniene, ${ }^{1,2}$ Gintaras Kuprionis ${ }^{3}$ \\ 'Endocrinology department, Hospital of Lithuanian, University of \\ Health Sciences Kauno klinikos, Lithuania \\ ${ }^{2}$ Encocrinology Department, Lithuanian University of Health \\ Sciences, Lithuania \\ ${ }^{3}$ Radiology department, Hospital of Lithuanian University of \\ Health Sciences Kauno klinikos, Lithuania
}

Correspondence: Diana Simoniene, Hospital of Lithuanian, University of Health Sciences Kauno klinikos, Lithuania, Tel +37061935361, Email simoniene.diana@gmail.com

Received: April 22, 2017 | Published: November 15, 2018
Abbreviations: MT, metastasis to thyroid; FNAC, fine needle aspiration cytology; TUS, thyroid ultrasound

\section{Introduction}

The thyroid gland is an uncommon site for metastasis. ${ }^{1}$ Clinically, it represents less than $4 \%$ of thyroid malignancies in clinical and surgical studies, despite that the thyroid gland is one of the most vascular organs of the body. ${ }^{2,3}$ It is proved when metastatic cancer to the thyroid occurs, and then the prognosis of overall survival is poor. ${ }^{4}$ In our patient, the time from diagnosis of a primary tumor to TM detection and death was only two months. The overall survival of patients with metastases to the thyroid is $33.7 \pm 6.6$ months. ${ }^{5} \mathrm{~A}$ history of cancer can be helpful in reaching the diagnosis; however, a final confirmation by histopathology is required. In this case report, we present a patient who developed a massive metastatic thyroid lesions of a primary non-small cell lung cancer and a rapid disease progress to death. Also, we review the clinicopathological features and management of secondary thyroid tumors.

\section{Case presentation}

A 58-year-old male, a prior smoker was diagnosed with non-small cell lung cancer T4N3M1 St IV. Histo pathological examination of the tumor confirmed infiltrative adenocarcinoma, G1. About eight months earlier, he was treated in Pulmonology-oncology clinic for an adenocarcinoma of the right upper lobe (LUL) of the lung with metastasis to right pleura; he received chemotherapy cisplatin and pemetrexed for four cycles. He had no response after four cycles, so his therapy was stopped as a case of lack of response to systemic therapy. The patient was left for observation. At the beginning of the disease, the patient was examined by the endocrinologist because of the presence of positron emission tomography (PET) positive thyroid gland nodule that was hyper metabolic. A fine needle aspiration (FNA) revealed a cystic degeneration. It was intended to repeat the FNA after 3-6 months. After two months of discontinuation of chemotherapy, the patient presented to our department with a three-week history of increasing volume of the neck, shortness of breath, and changes in voice and swallowing disorder. Physical examination revealed a chronic respiratory failure with tachypnea, tachycardia, and stridor. On palpation of the neck, a large-sized non-tender, firm mass with a nodular consistency was detected in the whole thyroid gland with cervical lymphadenopathy.

Initially, we thought about anaplastic thyroid cancer or lymphoma because of very rapid growth of thyroid mass. Thyroid ultrasound examination showed an enlarged thyroid gland; its structure was hypoechogenous and non-homogenous. Enlarged regional lymph nodes were also noted with obliteration of their ovoid morphology and loss of the echogenic hilum (Figure 1A). Further imaging evaluation with contrast-enhanced computed tomography of the neck revealed an irregular tumor mass, which infiltrated neck soft tissue and vessels, causing airway stenosis up to $1 \mathrm{~cm}$ in diameter. The image was similar to hypo pharyngeal cancer. Also, by reason of voice changes, he was reviewed by an otolaryngologist and diagnosed a paralysis of one side vocal cord. Biochemical testing of thyroid function showed subclinical hyperthyroidism. After 1 week, thyroid ultrasound was repeated: thyroid was dramatically increased in size with its hypoechogenous and non-homogenous structure. On both lobes it was observed some up to $18 \mathrm{~mm}$ hyperechogenous and isoechogenous mixed nodes with pathological regional lymph nodes (Figure 2B). Thyroid ultra sonography-guided fine-needle aspiration cytology (US-FNAC), cervical lymph nodes and laryngeal biopsies and particularly immunocytochemistry documented metastasis from primary lung cancer. Immunohistochemistry demonstrated that these cells were positive for TTF-1 and negative for thyroglobulin, consistent with metastatic disease from lung origin (Figure 3). The patient was deemed a nonsurgical candidate.

During several days in our department, the patient developed acute respiratory failure and for further treatment had to be shifted to Critical Care clinic; the patient underwent intubation and mechanical ventilation. Hypotension was corrected by vasopressors. Because of his bad overall performance status, further systemic therapy was not available. After nine days, the patient died. 


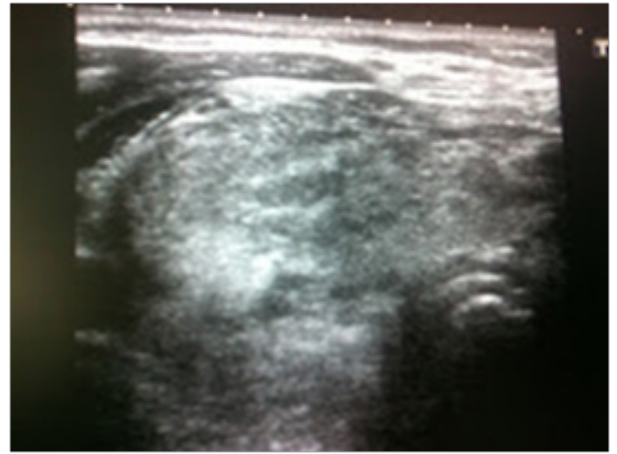

Figure IA Thyroid ultrasound showing enlarged thyroid gland with hypoechogenous and non-homogenous structure.

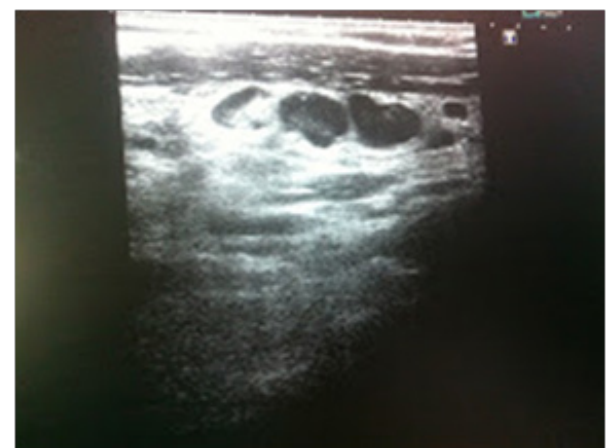

Figure IB Enlarged regional lymph nodes were also noted with obliteration of their ovoid morphology and loss of the echogenic hilum.

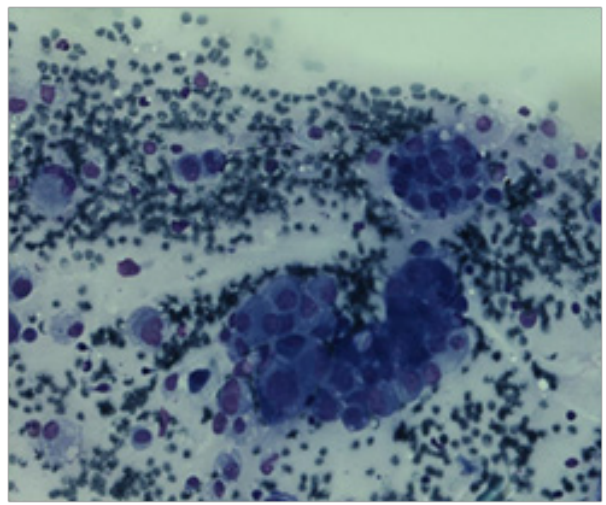

Figure 2 Immunohistochemistry demonstrating a malignant neoplasm of various-sized cells glandular in structure.

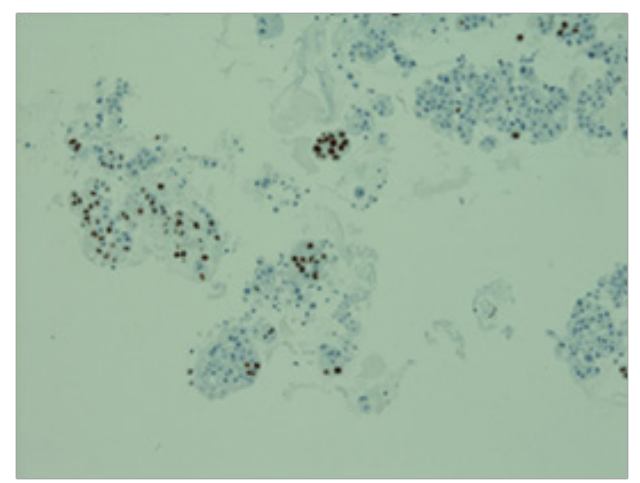

Figure 3 On immunocytochemistry, the malignant cells were focally positive to thyroid-transcription factor-I (TTF-I).

\section{Discussion}

Metastases to the thyroid gland are infrequent; TM represent between $1.15 \%$ and $3 \%$ of thyroid cancers. ${ }^{6}$ The most typical tumors metastasizing to the thyroid are the renal cell, breast, lung, esophageal and gastric carcinomas as well as melanoma. ${ }^{7}$ Among lung cancer types causing thyroid metastasis, adenocarcinomas are the commonest. ${ }^{8}$

Early detection and treatment of TM critically affect patient outcomes; therefore, differential diagnosis of thyroid lesions showing some ultrasound (US) features is essential. Ultrasound features that help discriminate malignant nodules from benign are the shape, margin, echogenicity, and a presence of calcification; tumor size depends on the diagnostic accuracy of ultrasound. ${ }^{9}$ US features of TM are known to be nonspecific, appearing as inhomogeneous, and hypo echoic, ill-defined, solid and nodular lesions with or without metastatic cervical lymphadenopathy. Solid composition, hypoechogenicity, non-circumscribed margins, no calcifications, parallel shape, and the presence of suspicious cervical lymph nodes are the most common US features in thyroid metastasis. ${ }^{10}$ According to Chaudhary, sonographically, metastases to thyroid gland present as a solitary or multiple hypo echoic homogeneous masses without calcification. ${ }^{11}$ While US features of thyroid metastases are nonspecific, but the presence of suspicious lymph nodes in a patient with anamnesis of cancer should give a suspicion of metastasis in thyroid masses.

Typically, the interval between the diagnosis of the primary tumor and the detection of thyroid metastasis is from one month to twentysix years. ${ }^{12}$ The report of another study revealed that thyroid metastasis appeared on average 48 months after discovery of the primary tumor. ${ }^{3}$ In this case report, the interval was eight months after the discovery of the lung cancer, and only two months from the first symptoms of TM till the patient's death. According to Papi 10-year-experience study, the mean time from thyroid metastasis (TM) detection to patients' death and between patients who underwent thyroidectomy was 14 months versus the patients without surgery treatment of TM was 4 months $(p=0,002)$. While, the mean survival time in patients with TM between these two groups did not significantly differ. ${ }^{13}$ Another study showed the median survival from surgery of TM to death or last follow-up was 26.5 (range, 2-114) months. The study concluded that in patients with $\mathrm{TM}$, local control of metastatic disease can be successfully achieved with surgical resection. ${ }^{14}$ Moreover, adolescents and young adults who diagnosed a thyroid metastasis have a significantly greater risk of mortality than those with primary thyroid cancer. These patients are than 6.6-times more likely to die than patients with primary cancer. ${ }^{14}$ Finally, detection of metastasis to the thyroid gland often indicates the poor survival prognosis and aggressive surgical and medical therapy may be effective just in a small percentage of patients. ${ }^{4}$ In case of multiple metastases, the overall survival is $5 \%$ in 5 years. ${ }^{15}$

The distinction between primary and secondary malignant thyroid tumors is difficult because metastatic thyroid tumors are rarely clinically noticeable. ${ }^{16}$ In our opinion, the reason for such a phenomenon is primarily the rapid development of primary cancer and an unusual site for metastasis. ${ }^{17}$ In our case, cystic degeneration in FNA resulted in misdiagnosis. We thought that in this case, the thyroid nodules were benign, but the rest thyroid tissue was involved by diffuse pulmonary metastasis. It is just a theoretical idea, because autopsy was not performed for this patient. However, the fast cancer growth and later biopsy support this hypothesis. As a consequence, 
the decision to perform thyroidectomy was not made. Delayed diagnosis of the thyroid metastasis was one of the main reasons for the short survival period. Another reason was a very rapid primary and simultaneously metastatic cancer progression.

In conclusion, a diagnosis of metastatic disease should be treated as soon as possible because it may prolong patient's life.

\section{Acknowledgements}

None.

\section{Conflict of interest}

The author declares no conflict of interest.

\section{References}

1. Yang M, Wang $\mathrm{W}$, Zhang $\mathrm{C}$. Thyroid gland metastasis arising from breast cancer: A case report. Oncol Lett. 2013;5(6):1836-1838.

2. Namad T, Wang J, Shipley R, et al. Thyroid metastasis from nonsmall cell lung cancer. J Case Rep Oncol Med. 2013;2013:208213.

3. Chung SY, Kim EK, Kim JH, et al. Sonographic findings of metastatic disease to the thyroid. Yonsei Med J. 2001;42:411-417.

4. Nakhjavani MK, Gharib H, Goellner JR, et al. Metastasis to the thyroid gland. A report of 43 cases. J Cancer. 1997;79(3):574-578.

5. Nixon IJ, Whitcher M, Glick J, et al. Surgical management of metastases to the thyroid gland. J Ann SurgOncol. 2011;18(3):800-804.

6. Martin C, Touré E, Dehesdin D, et al. Thyroid metastasis from cardia cancer. J Otorhinolarygol. 2014;3:5.

7. Cichon S, Anielski R, Konturek A, et al. Metastases to the thyroid gland: Seventeen cases operated on in a single clinical center. $J$ Langenbecks Arch Surg. 2006;391:581-587.
8. Lin JD, Weng HF, Ho YS. Clinical and pathological characteristics of secondary thyroid cancer. Thyroid. 1998;8(2):149-153.

9. Moon WJ, Jung SL, Lee JH, et al. Benign and malignant thyroid nodules: US differentiation--multicenter retrospective study. $J$ Radiology. 2008;247(3):762-770.

10. Yoon JH, Kim EK, Kwak JY, et al. Sonographic features and ultra sonography-guided fine-needle aspiration of metastases to the thyroid gland. Ultrasonography. 2014;33(1):40-48.

11. Chaudhary V, Bano S. Thyroid ultrasound. Indian J Endocrinol Metab. 2013;17(2):219-227.

12. Menegaux F, Chigot JP. Thyroid metastases. Ann Chir. 2001;126(10):981984.

13. Papi G, Fadda G, Corsello SM, et al. Metastases to the thyroid gland: Prevalence, clinicopathological aspects and prognosis: A 10-year experience. Clin Endocrinol (Oxf). 2007;66(4):565-571.

14. Goldfarb M, Freyer DR. Comparison of secondary and primary thyroid cancer in adolescents and young adults. $J$ Cancer. 2014;120(8):11551161.

15. Heffess CS, Wenig BM, Thompson LD. Metastatic renal cell carcinoma to the thyroid gland: A clinicopathologic study of 36 cases. Cancer. 2002;95(9):1869-1878.

16. Mirallie E, Rigaud J, Mathonnet M, et al. Management and prognosis of metastases to the thyroid gland. J Am Coll Surg. 2005;200(2):203-207.

17. Katsenos S, Archondakis S, Vaias M, et al. Thyroid gland metastasis from small cell lung cancer: An unusual site of metastatic spread. J Thorac Dis. 2013;5(2):E21-4. 